\title{
Comparative Study of Blood Culture with Rapid Diagnostic Tests for Diagnosis of Enteric Fever in a Tertiary Centre of NE-India
}

\author{
Anjan Sarma', Dipa Barkataki ${ }^{2}$ \\ 'Department of Microbiology, FAAMCH Barpeta, Assam \\ 2Department of Microbiology, SMCH, Assam
}

\section{A B S T R A C T}

\begin{abstract}
Introduction: Enteric fever is a global health problem and is also endemic in India. The laboratory diagnosis of enteric fever is very important mainly because in post-antibiotic era most cases are treated empirically and investigated for various causes of PUO including enteric fever. A fast, reliable, and easy to perform serodiagnostic test with a higher sensitivity and specificity is required for rapid diagnosis and management of typhoid cases, thereby enabling clinicians to initiate an early therapy, reducing morbidity and its complications.

Aim: The study was performed to evaluate the performance of Widal test and Typhidot, a lateral flow immunoassay test.

Materials and Methods: This study was carried out in the Department of Microbiology in a tertiary care center for 1 year (August 2016 July2017). A total of 110 blood samples were collected from patients (cases) clinically suspected of enteric fever. Culture and serological tests were done in all the 110 cases. Salmonella isolation was done by conventional methods of culture. Demonstration of presence of antibodies were done by Widal tests using commercially available kits. Typhidot, a lateral flow immunoassay, was used to test and detect lgM and lgG antibodies against the outer membrane protein (OMP) of the Salmonella Typhi. The performances of the tests were compared, and diagnostic accuracy determined.

Results: Out of 110 cases, highest percentage of positive cases were found by Typhidot test (29.09\%) followed by Widal test (23.63\%). $18.18 \%$ cases were confirmed by blood culture. Taking blood culture as gold standard, it was found that Widal test had a sensitivity of $30 \%$ and specificity of $78 \%$ and Typhidot had a sensitivity of $75 \%$ and specificity of $81 \%$.

Conclusion: Though definitive diagnosis of enteric fever is isolation of the causative organism from the patient, Widal test and Typhidot test are important diagnostic tools in the medical set up. Typhidot is fast becoming a practical alternative to Widal test. Typhidot test had a fairly good sensitivity and specificity, whereas Widal test had good specificity but a lower sensitivity.

Keywords: Blood culture, Widal, Typhidot, Sensitivity, Specificity

JMS: 2019; 22(3):21-26ＤOI: https://doi.org/10.33883/jms.v22i3.474
\end{abstract}

\section{INTRODUCTION}

Enteric fever is a global health problem and is also endemic in India. It is a systemic disease characterized by fever and abdominal pain and caused by dissemination of S.Typhi or S.Paratyphi. The etiologic agents of enteric fever i.e S.Typhi and S.Paratyphi serotypes A, B, and Chave no known hosts other than humans. Most commonly, food-borne or waterborne transmission results from fecal contamination by ill or asymptomatic chronic carriers. Health care workers occasionally acquire enteric fever after exposure to infected patients or during processing of clinical specimens and

\begin{tabular}{|l|l|}
\hline & Access this article online \\
Website: & \\
WOI.jmsskims.org
\end{tabular}

cultures. ${ }^{[1]}$

The laboratory diagnosis of enteric fever is very important mainly because most of the patients are treated empirically by the local medical practitioners and when the fever does not subside, these cases are labeled as pyrexia of unknown

Correspondence:

Dr. Anjan Sarma, M.D

Senior Resident, Department of Microbiology, FAAMCH, Barpeta, Assam

Email:juman4u16@gmail.com

How to cite this article: Sarma A, Barkataki D. Comparative Study Of Blood Culture with Rapid Diagnostic Tests for Diagnosis Of Enteric Fever In A Tertiary Centre Of NE-India. jms 2019;22(3):21-26

Received:07-07-2019 Accepted: 13-12-2019 Published: 
Sarma A; et al; Comparative Study of Blood Culture with Rapid Diagnostic Tests for Diagnosis

origin (PUO) and investigated for various causes of PUO including enteric fever. At this stage the typical signs and symptoms are hardly observed. ${ }^{[2]}$

The presence of S.Typhi or S.Paratyphi is detected either by culture of the organism or by the demonstration of specific antibodies or antigen in the serum ${ }^{[3]}$. Blood culture is a more practical and sensitive method. However, it is not always available and, when available, it takes around $24-48 \mathrm{hrs}$. As a result, diagnosis may be delayed or overlooked and patients without typhoid fever may receive unnecessary and inappropriate antimicrobial treatment. For this reason, in developing countries typhoid rapid antibody tests can facilitate diagnosis and disease management. ${ }^{[4]}$

The World Health Organization (WHO) has issued no recommendations on the use of typhoid rapid antibody tests. Accurate diagnostics for typhoid fever could provide valuable diagnostic information for patient management and make it possible to estimate the incidence of typhoid fever in low-resource settings. ${ }^{[4]}$

Sero-diagnosis depends upon the 100-year-old Widal test, and other serological diagnostic tools which have limitations because of their low sensitivity and/or specificity. There is an urgent need for the rational design and evaluation of effective and appropriate diagnostic tools for enteric fever which must include the emerging threat of S. Paratyphi $\cdot{ }^{[5]}$ A fast, reliable, and easy to perform serodiagnostic test with a higher sensitivity and specificity is required for rapid diagnosis and management of typhoid cases, thereby enabling clinicians to initiate an early therapy, reducing morbidity and its complications.

This study was undertaken to systematically evaluate the utility of rapid diagnostic tests such as Widal and Typhidot by comparing the diagnostic accuracy of these tests with isolation of the organism by blood culture in terms of sensitivity and specificity. ${ }^{[6}$

\section{MATERIALS \& METHODS}

This study was carried out in the Department of Microbiology, Gauhati Medical College Hospital, Guwahati for a duration of one year from August'2016-July' 2017 after obtaining ethical clearance from the Institutional Ethical Committee and consent from the patients.

A total of 110 blood samples were collected from patients (cases) clinically suspected of enteric fever.

\section{Inclusion Criteria:}

1) Patients attending outpatient department and inpatients of various departments clinically suspected to be suffering from enteric fever were included in the study.

2) Patients suspected of having enteric (typhoid) fever may have the following signs \& symptoms:

- Gradual onset fever with headache, malaise, anorexia, a coated tongue and abdominal discomfort with either constipation or diarrhea.

- Step-ladder pyrexia, with relative bradycardia and toxemia.

- A soft, palpable spleen with hepatomegaly.

- Rose-spots that fade on pressure (mainly appear during 2 nd or 3 rd week of enteric fever).

3) Individuals of both sexes representing all ages.

\section{Exclusion Criteria}

1) Patients having no signs \& symptoms of enteric fever.

2) Fever with any obvious focus for other infection such as urinary tract infection, blood septicaemia, fungal infection, otitis media etc.

3) Patients with prior antibiotic administration and $\mathrm{TAB}$ vaccination.

For each patient, blood samples were collected under aseptic conditions and immediately inoculated into appropriate media. The specimen was collected on the $1^{\text {st }}$ day of their attendance or admission in the department before any medication was started. Culture and serological tests were done in all the 110 cases.

$10 \mathrm{ml}$ of blood from adults and 5-7 $\mathrm{ml}$ of blood from paediatric age group were collected aseptically from the antecubital vein of the forearm. 5-6 $\mathrm{ml}$ of blood was inoculated into BHI blood culture bottles and mixed thoroughly by rotation. The remaining blood was transferred to a sterile clot vial and allowed to clot. The serum was used for serological tests.

Salmonella isolation was done by conventional methods of culture. Species identification was done by commercially available test kit KB011 HiSalmonella (Hi Media) according to manufacturer's literature. Each KB011 kit is a standardized colorimetric identification system using 7 
Sarma A; et al; Comparative Study of Blood Culture with Rapid Diagnostic Tests for Diagnosis

conventional biochemical tests and 5 carbohydrate utilization tests.

Confirmed Salmonella isolates were tested by slide agglutination test using commercially prepared anti-sera using $\mathrm{O}$ typing sera for group identification and $\mathrm{H}$ typing sera for type identification.

Demonstration of presence of anitibodies was done by Widal test using commercially available kits (Tulip diagnostics Ltd). Widal test was performed by semiquantitative tube method using different antigens such as $\mathrm{S}$. Typhi O (TO), S. Typhi H (TH), S. Paratyphi A H (AH), and S. Paratyphi B H (BH). Tube titres of 1:80 and above were taken as diagnostically significant.

Typhidot kit (OnSite Typhoid IgG/IgM Rapid Test-Cassette from CTK Biotech, Inc) was used to test and detect IgM and IgG antibodies against the outer membrane protein (OMP) of the Salmonella Typhi. It is a lateral flow immunoassay for the simultaneous detection and differentiation of antiSalmonella Typhi (S. Typhi) IgG and IgM in human serum or plasma. The test was performed according to the manufacturer's instructions, and reading was taken after observing the control line showing the test to be valid. All data were entered in Excel spreadsheet (Microsoft, USA) and analysis was done. The performances of the tests were compared, and diagnostic accuracy (sensitivity, specificity, positive predictive value and negative predictive value) of these tests were calculated.

\section{RESULTS \& OBSERVATIONS}

Out of 110 cases, 75 cases were male and 35 were female. Majority of the cases (45.45\%) belong to the age group of 21-40 years with overall male-female ratio 2.14: 1 . Majority of the male cases $46.67 \%$ (35/75) \& female cases $42.85 \%$ (15/35) belonged to age group of 21-40years.

Out of 110 cases, highest percentage of positive cases were found by Typhidot (29.09\%) followed by Widal (23.63\%). $18.18 \%$ cases were confirmed by blood culture.

Table 1: Results of Cultures \& serological tests

\begin{tabular}{|c|c|c|c|}
\hline Investigations & Total & Positive & Percentages \\
\hline Blood Culture & 110 & 20 & $\mathbf{1 8 . 1 8 \%}$ \\
\hline Widal Test & 110 & 26 & $\mathbf{2 3 . 6 3 \%}$ \\
\hline Typhidot for IgG/IgM & 110 & 32 & $\mathbf{2 9 . 0 9 \%}$ \\
\hline
\end{tabular}

Graph 1: Age \& sex distribution of total cases

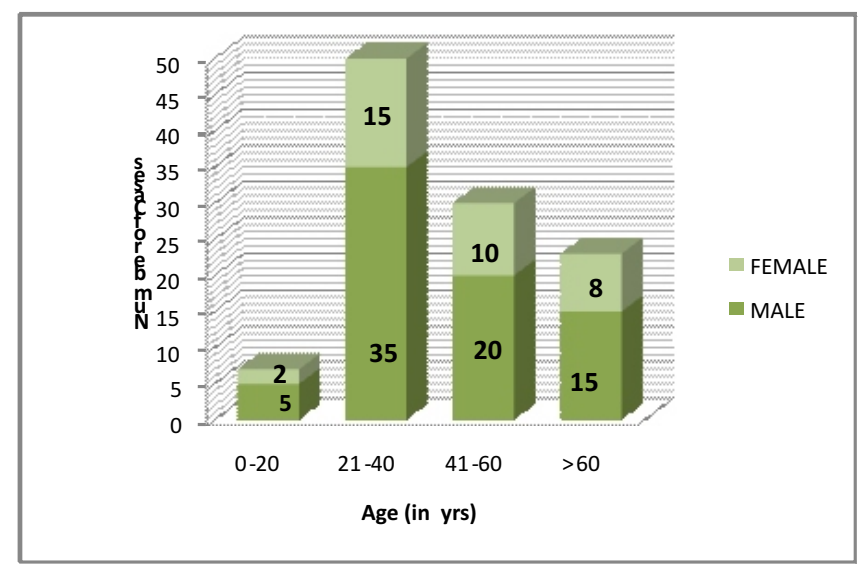

54 cases were found positive to have enteric fever on the basis of isolation of the organism in 20 cases, a positive Widal test (diagnostic titre) in 26 cases and a positive Typhidot antibody test in 32 cases as shown in the table below.

Table 2: Distribution of Positive cases

\begin{tabular}{|c|c|c|}
\hline Combination of Tests & $\begin{array}{c}\text { No. of positive } \\
\text { cases/110 } \\
\text { cases }\end{array}$ & $\begin{array}{c}\text { Percentages } \\
\text { (positive) }\end{array}$ \\
\hline $\begin{array}{c}\text { Blood Culture }+ \\
\text { Typhidot }\end{array}$ & 14 & $25.92 \%$ \\
\hline Blood Culture + Widal & 5 & $09.26 \%$ \\
\hline $\begin{array}{c}\text { Blood Culture + } \\
\text { Widal+Typhidot }\end{array}$ & 1 & $01.85 \%$ \\
\hline Widal + Typhidot & 3 & $05.55 \%$ \\
\hline Only Widal test & 17 & $31.48 \%$ \\
\hline Only Typhidot test & 14 & $25.92 \%$ \\
\hline Total & $\mathbf{5 4 / 1 1 0}$ & $\mathbf{4 4 . 5 4 \%}$ \\
\hline
\end{tabular}

$70.37 \%$ (38 cases) of the positive cases either by bacteriological or/and serological tests were male and $29.63 \%$ were females ( 16 cases), with a male and female ratio $2.4: 1$. $44 \%$ of suspected enteric fever cases came during the $1^{\text {st }}$ week of illness followed by $2^{\text {nd }}$ week $(36 \%)$ and $3^{\text {rd }}$ week $(20 \%)$. Majority of blood culture positive (75\%) and typhidot test positive (53\%) cases were detected in the $1^{\text {st }}$ week, whereas Widal test (69\%) was found to be positive mostly in the $2^{\text {nd }}$ week and a few in the $3^{\text {rd }}$ week of illness. Majority of the cases were during the rainy season of April to July.

Using blood culture as the standard and reference test for diagnosis of enteric fever, the sensitivity and specificity of the Widal and Typhidot tests were calculated from the 
Sarma A; et al; Comparative Study of Blood Culture with Rapid Diagnostic Tests for Diagnosis

samples having growth of Salmonella species as true positives and samples with no growth or growth of other organisms as true negatives.

It was found that Typhidot has a high sensitivity of $75 \%$ and specificity of $81 \%$ while Widal test has a low sensitivity of $30 \%$ and a high specificity of $78 \%$. (Table 3 )

Table 3: The performance of the different tests for diagnosis of enteric fever

\begin{tabular}{|l|c|c|c|c|}
\hline Test & Sensitivity & Specificity & $\begin{array}{c}\text { Positive } \\
\text { predictive } \\
\text { value }\end{array}$ & $\begin{array}{c}\text { Negative } \\
\text { predicti } \\
\text { ve value }\end{array}$ \\
\hline Widal & $30 \%$ & $78 \%$ & $23 \%$ & $83 \%$ \\
\hline Typhidot & $75 \%$ & $81 \%$ & $47 \%$ & $93 \%$ \\
\hline
\end{tabular}

\section{DISCUSSION}

The diagnosis of enteric fever currently depends on the isolation of Salmonella from a patient, most commonly by blood culture. This facility is not available in many areas where the disease is endemic. The other method is PCRbased amplification of DNA from the blood of enteric fever patients, but this technique requires expertise and a wellequipped laboratory. Antigen detection has not been investigated much and detecting an immune response specific for typhoid fever has been done only with antibody detection. ${ }^{[s]}$

In this present study, out of 110 cases, 18 cases $(16.3 \%)$ of S.Typhi and 2 cases (1.8\%) of S.Paratyphi were identified with total prevalence of typhoid fever by blood culture at $18.18 \%$. Similar observation was seen in studies done by K. K Patel et al (2013) ${ }^{[7]}$ and P Bhattacharya et al (2017) ${ }^{[8]}$ who reported $16.6 \%$ and $16.4 \%$ blood culture positivity respectively. Relative low rate of isolation from blood culture in some studies has been attributed to delay in visiting the physician for diagnosis, wide spread antibiotic use and the difficulties of obtaining large enough blood volumes.

In the present study, bacteriological and serological assessment of 110 clinically suspected cases of enteric fever was done. Maximum culture positive (55\%) and seropositive cases $(47.06 \%)$ were observed in the age group 2140 yrs. Overall, positive cases confirmed either by bacteriological and/or serological is also maximum (50\%) in the age group 21-40 yrs. This correlates with the study done by of J Sharma et al (2013) ${ }^{[9]}$ who observed maximum number of typhoid positive cases in between ages 21-40 yrs though prevalence was high in all age and sex group suggesting lack of immunity. The possible causes for enteric fever being common in age group 21-40 yrs include their mobility, consumption of unhygienic food outside home and drinking of contaminated or unfiltered water in colleges and offices or at their jobs.

Majority of the 110 cases in the present study were males (75) with male-female ratio 2.14:1 and 14 cases out of the 20 blood culture positive cases were male and 6 cases were female with male-female ratio of 2.33:1. Sero-positivity was also observed to be higher in males than females. This correlates with the studies of SC Sood and PN Taneja ${ }^{[10]}$ and SN Khosla et al ${ }^{[11]}$. High male preponderance was also observed by S Jog et al (2008) ${ }^{[12]}$ who reported male-female ratio of 1.64:1. This might be due to our cultural background where male is more likely to report to hospital, at the same time more likely to contract infection due to more outdoor activities.

In this present study, out of 110 cases, Widal test was found positive in 26 cases $(23.63 \%)$ which correlates with study done by J Sharma et al (2013) ${ }^{[9]}$ who reported Widal test positivity of $26.2 \%$, S Udayakumar et al $(2017)^{[13]}$ of $39.6 \%$ and Enabulele et al $(2016)^{[14]}$ of $45.76 \%$. In the present study, sensitivity of Widal test was found to be $30 \%$ which correlates with study done by Enabulele et al $(2016)^{[14]}$ who reported 35\%. Specificity of Widal test was found to be 78 $\%$ which correlates with observation seen in study done by Udayakumar et al ${ }^{[13]}(79.3 \%)$ and $\mathrm{G}$ Andualem et $\mathrm{al}^{\left[{ }^{[15]}\right.}$ (68.44\%). In this study, positive predictive value of Widal test was found to be $23 \%$ and Negative predictive value of $83 \%$. The role of Widal test is controversial, because the sensitivity, specificity, and predictive values of this widely used test vary considerably among geographic areas. Sharing of O \& H antigen by other Salmonella serotypes and other members of Enterobacteriaceae makes the role of Widal test less specific, and hence, its use is controversial in diagnosis of enteric fever. It is possible that the Widal test would have performed better if paired sera were tested to demonstrate the rising titers. Patients rarely return for outpatient follow-up once treated so that obtaining paired sera in a routine clinical setting is unlikely, and hence clinicians widely rely on "positive" Widal test done on a 
Sarma A; et al; Comparative Study of Blood Culture with Rapid Diagnostic Tests for Diagnosis

single serum sample.

In this present study, Typhidot test positivity was found to be $29.09 \%$ ( 32 cases) which correlates with study done by KJ Prasad et al $(2015)^{[16]}$ at $36.24 \%$ followed by Khalid Mehmood et al (2015) ${ }^{[17]}$ at $37.24 \%$. Sensitivity of Typhidot was found to be $75 \%$ which correlates with study of $\mathbf{S}$ Udayakumar et al (2017) ${ }^{[13]}$ at $81.7 \%$. In this study, positive predictive value of Typhidot test was found to be $47 \%$ and negative predictive value at $93 \%$. Typhidot meets one of the criteria of an ideal diagnostic test as it doesn't usually miss the diagnosis when compared to blood culture. Only 5 cases, which were blood culture positive, were negative by Typhidot. All these 5 cases presented with duration of fever more than 10 days. Typhidot is a simple, rapid diagnostic test available commercially, requiring less than $30 \mathrm{~min}$, and minimal training. Typhidot test appears to be useful alternative to Widal as it is more specific and useful in early diagnosis of enteric fever. Typhidot appears to have slight advantage over Widal test in that results are rapid, can detect the disease early in the 1 st week and is easy to perform.

\section{CONCLUSION}

From the present study, it could be concluded that the clinical diagnosis of enteric fever is a true diagnostic challenge as it may simulate many fever causing infectious diseases. Though definitive diagnosis is isolation of the causative organism from the patient, Widal test and Typhidot test are important diagnostic tools in the medical set up. Typhidot is fast becoming a practical alternative to Widal test in the diagnosis of Typhoid fever on account of its increased sensitivity, early detection of cases, and ease of procedure with minimal infrastructure and availability of results on the same day. Although both Widal and Typhidot tests can be used to suspect enteric fever more strongly than on clinical grounds alone, it is prudent to follow them up with blood culture confirmation.

\section{REFERENCES}

1. Kasper DL, Fauci AS, Hauser S, et al, editors. Harrison's principles of internal medicine, 19th ed. New York: The McGraw-Hill Companies, Inc.; 2015

2. Singh S. Pathogenesis and laboratory diagnosis. Journal, Indian Academy of Clinical Medicine. 2001;2.: 16-20

3. Collee JG, Duguid JP, Fraser AG, Marmion BP,
Simmons A. Salmonella. Mackie and McCartney practical medical microbiology. 1996;14:385-404

4. Keddy KH, Sooka A, Letsoalo ME, Hoyland G, Chaignat CL, Morrissey AB, Crump JA. Sensitivity and specificity of typhoid fever rapid antibody tests for laboratory diagnosis at two sub-Saharan African sites. Bulletin of the World Health Organization. 2011; 89:640-7.

5. Wain J, Hosoglu S (2008) The laboratory diagnosis of enteric fever. J Infect Dev Ctries 2:421-425. doi: 10.3855/jidc. 155

6. Yadav K, Yadav SK, Parihar G. A Comparative Study of typhidot and widal test for Rapid Diagnosis of Typhoid Fever. Int. J. Curr. Microbiol. App. Sci. 2015;4(5):34-8.

7. K. K Patel, D.Majumdar, Sarita Patel, R.Sujatha, D.N. Singh. Emerging Ciprofloxacin and Multi Drug Resistant Salmonella Species Isolated from Patients with Enteric Fever in Chhattisgarh. Journal of Evolution of Medical and Dental Sciences 2013; Vol 2, Issue 11, March 18; Page: 1638-1642

8. Bhattacharya P, Saha BK, Paul UK, Bandyopadhyay A. Blood Culture in Clinically Suspected Typhoid Fever. Int J Sci Stud 2017;4(11):53-56.

9. Sharma J, Malakar M. Distribution of Typhoid fever in different rural and urban areas of Lakhimpur District of Assam. International journal of research and development of health. 2013;1(3):109-14.

10. Sood SC, Taneja PN. Typhoid Fever. Clinical Picture and Diagnosis. Indian Journal of Child Health. 1961;10(2):69-76.

11. Khosla SN, Miglani S, Sabharwal U, Khosla A. Incidence of carrier state in treated patients of typhoid. The Journal of the Association of Physicians of India. 1995 Mar; 43(3):189-90.

12. Jog S, Soman R, Singhal T, Rodrigues C, Mehta A, Dastur FD. Enteric fever in Mumbaiclinical profile, sensitivity patterns and response to antimicrobials. JAPI. 2008 Apr; 56:237-40.

13. Udayakumar, S., Pushpalatha, K., Sagar, H. M., Swathi, M., Yoganand, R., \& Sushma, C. (2017). Comparative study of Typhidot-M with Widal and blood culture in diagnosis of enteric fever. Indian Journal of Child Health, 4(1), 64-67. 
Sarma A; et al; Comparative Study of Blood Culture with Rapid Diagnostic Tests for Diagnosis

14. Enabulele O, Awunor SN. Typhoid fever in a Tertiary Hospital in Nigeria: Another look at the Widal agglutination test as a preferred option for diagnosis. Nigerian medical journal: journal of the Nigeria Medical Association. 2016 May; 57(3):145-149.

15. Andualem G, Abebe T, Kebede N, Gebre-Selassie S, Mihret A, Alemayehu H. A comparative study of Widal test with blood culture in the diagnosis of typhoid fever in febrile patients. BMC research notes. 2014 Dec; 7(1):653.
16. Prasad KJ, Oberoi JK, Goel N, Wattal C. Comparative evaluation of two rapid Salmonella-IgM tests and blood culture in the diagnosis of enteric fever. Indian journal of medical microbiology. 2015 Apr 1;33(2):237-42.

17. Mehmood K, Sundus A, Naqvi IH, Ibrahim MF, Siddique O, Ibrahim NF. Typhidot-A blessing or a menace. Pakistan journal of medical sciences. 2015 Mar; 31(2):439-43. 